\title{
Research on Logistics Issues in Equipment Autonomic Support
}

\author{
Yongle $\mathrm{He}^{1,}$ a, $\mathrm{Na} \mathrm{Lin}{ }^{2, b,}{ }^{*}$, Jingtao Shang ${ }^{1, \mathrm{c}}$ \\ ${ }^{1}$ China North Vehicle Research Institute, Beijing 100072, China; \\ ${ }^{2}$ School of Mechanical Engineering, Dalian University of Technology, Dalian 116024, China. \\ aheyongle2008@163.com, b, *linnax7@gmail.com, 'shang850826@163.com
}

\begin{abstract}
This paper studies the typical logistics problem in equipment support, i.e. the decisionmaking optimization of material supply, which refers specifically to the transportation and distribution of materials and equipment (such as ammunition, oil, personnel, medical supplies, maintenance accessories, maintenance equipment, etc.) during the replenishment process. Firstly, we model the problem as a multi-depot vehicle routing problem (MDVRP), and then design a two-stage heuristic algorithm to solve it. We construct an example of 150 demand points and 3 resource points to validate the effectiveness of the algorithm, and compare the two-stage heuristic algorithm with the simulated annealing-based particle swarm optimization (SAPSO) algorithm. The experimental results show that the two-stage heuristic algorithm can better solve the logistics problem in equipment autonomic support.
\end{abstract}

Keywords: equipment autonomous support; material distribution; two-stage heuristic algorithm (TSHA); hybrid genetic algorithm (HGA).

\section{Introduction}

Equipment support is a general term for all kinds of technical measures and related support activities adopted to maintain and restore the good technical performance of weapons and equipment $[1,2]$. The autonomous support mode starts the maintenance support behavior at an appropriate time by fully utilizing the real-time status assessment of equipment, fault prediction information, and the network coordination ability of maintenance support resources, reducing equipment support costs and failure risks, improving equipment availability and mission success [3]. A typical logistics problem in equipment independent support is equipment distribution, which is essentially an MDVRP problem.

MDVRP is a variant of VRP, which belong to classical combinatorial optimization problem and first proposed by Dantzing and Ramser in 1959. For decades, researchers have done a lot of studies on VRP issues. The approaches to solving VRP are mainly classified into two aspects: exact method and heuristics. The exact method can often get the global optimal solution. However, it is not used widely because of the low efficiency in the big scale problem, which means the number of the customer is more than 50. On the contrary, heuristics can be efficient in big scale problem and give an approximate solution. State of the art heuristics include: particle swarm optimization (PSO) $[4,5]$ ant colony optimization (ACO) [6,7], genetic algorithm (GA) [8], simulated annealing (SA) $[9,10]$ and tabu search (TS) [11, 12], and each kind of heuristics have its own advantages to solve VRP. Comparing to others, the superiority of GA is that it has a strong robustness in solving complicated problem, which make it a popular algorithm in the practice.

\section{Problem Description}

Equipment support resource scheduling is a multi-objective complex integrated scheduling process that uses natural number sequence coding according to resource scheduling tasks. Let $V=\left\{V_{i} \mid i=1,2, \ldots, K, K \geq 2\right\}$ be the set of the vertex and graph $G=(V, A)$, where $R=\left\{R_{m} \mid m=1,2, \ldots, M, M \in\{1, N-1\}\right\}$ denotes the set of the request nodes and $S=\left\{S_{n} \mid n=N, \ldots, K, N \in\{2, K\}\right\}$ denotes the resource points. The resource scheduling problem of equipment support can be described as follows: equipment support is provided by $M$ resource points 
$R_{m}$ to $N$ demand points $S_{n}$, the storage capacity of $E_{v}$ for equipment at the M resource point is $P_{m v}$, and the demand for $E_{v}$ at the $\mathrm{N}$ demand point is $N_{n v}$; vehicle speed on road AIJ is related to road grade and road obstruction. The objective is to obtain the highest overall benefits of equipment support under the constraint conditions, and allocate the materials in $R_{m}$ to $S_{n}$.

$$
F(X)=\left\{\begin{array}{l}
\min f_{1}(X)=\sum_{m=1}^{M} \sum_{n=N}^{K} \sum_{i=1}^{L} \frac{p_{m n i} a_{i}^{l}\left(1+\frac{a_{i}^{j}}{1-a_{i}^{j}}+\frac{a_{i}^{q}}{1-a_{i}^{q}}\right)+\sum_{m=1}^{M} \sum_{n=N}^{K} \sum_{v=1}^{E} x_{m n v} a_{v}^{z}\left(1+\left(1-b_{m}\right)\right)}{\min f_{2}(X)=\sum_{m=1}^{M} \sum_{n=N}^{K} \sum_{i=1}^{L} \sum_{v=1}^{E} \frac{p_{m n i} x_{m n v} a_{i}^{L} a_{i}^{f}}{a_{v}}+\sum_{m=1}^{M} \sum_{n=N}^{K} \sum_{v=1}^{E} x_{m n v} a_{v}^{c}} \\
\qquad \text { s.t. }\left\{\begin{array}{l}
R_{m} \in\left\{R_{m}^{1} \cup R_{m}^{2} \cup R_{m}^{3} \cup R_{m}^{4}\right\} \\
\sum_{n=N}^{K} x_{m n v} \leq P_{m v} \\
\sum_{m=1}^{M} x_{m n v} \leq N_{n v} \\
T_{n 1} \leq t_{m n} \leq T_{n 2} \\
a_{i}=\left(v_{k}, v_{h}\right), p_{m n i} \in\{0,1\} \\
P_{m v} \geq 0, N_{n v} \geq 0, x_{m n v} \geq 0
\end{array}\right.
\end{array}\right.
$$

$f_{1}(X)$ is the time function of equipment supply, the symbolic meanings in the formulas are as follows: $P_{m n i} \in\{0,1\}$ denotes whether the $R_{m}$ to $S_{n}$ passes through the road $a_{j}$ or not, and $a_{i}^{l}$ is the length of $a_{j} ; a_{i}^{v}$ is the average speed of vehicles on $a_{j} a_{i}^{j}$ and $a_{i}^{q}$ are influence factors of traffic jam and driving speed of vehicles under weather conditions, respectively; $x_{m n v}$ is the quantity of $E_{v}$ supplied to $S_{n}$ by $R_{m} ; a_{v}^{z}$ is the unit loading time of $E_{v} ; b_{m}$ is the support ability level of $R_{m}$.

$f_{2}(X)$ is the cost function of equipment support, the symbolic meanings in the formula are: $a_{i}^{f}$ is the cost per unit distance for vehicle in $a_{j} ; a_{v}$ is the loading amount for $E_{v}$ and $a_{v}^{c}$ is the loading cost per unit number of $E_{v}$.

\section{The Proposed TSHA}

First, the resource points are allocated to demand points by distance based partitioning method, so that MDVRP can be converted to VRP. Then a hybrid genetic algorithm (HGA) is used to solve the path planning problem in equipment allocation decision-making, which includes five basic steps: (a) problem representation, (b) population initialization, (c) selection, (d) crossover, (e) mutation.

\subsection{The Allocation of the Request Points}

Firstly, the distances between the request points and all resource points are calculated, and the demand points are divided into the nearest resource points, so that the MDVRP problem can be transformed into the VRP problem.

\subsection{The Routing Problems in a Single Resource Point}

\subsubsection{Problem Representation}

A resource point will allocate more than one vehicle for equipment allocation, so the solution of the problem includes multiple sub-paths. We use the representation of sub-paths divided by separators. As shown in figure 1, there are three vehicles for equipment allocation. A complete solution is divided into three sub-paths: path 1:0-2-4-5-0, path 2:0-3-7-1-0, path 3:0-6-8-0. 


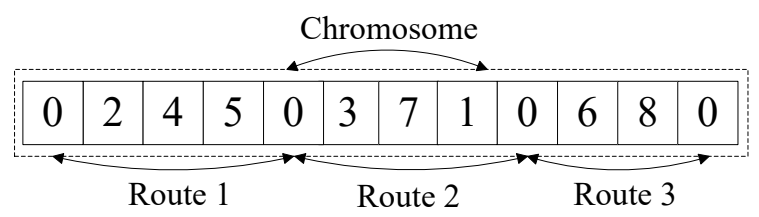

Fig.1 The problem representation of the material allocation in a single resource point

\subsubsection{The Initialisation of the HGA.}

We use scanning algorithm and random strategy to initialize population. That is to say, part of the initial part is generated by the scanning algorithm, and the rest is generated randomly. The former produces structural solutions to accelerate convergence, while the latter guarantees population diversity. Because structured initial solutions tend to reduce the diversity of population and cause premature convergence, random individuals should be the majority. After many attempts, we set the scale parameter to $9: 1$, that is, only $10 \%$ of the individuals generated by the scanning algorithm.

\subsubsection{The Selection of the HGA}

We use championship selection as the selection strategy of HGA, in which two individuals compete randomly and those with larger fitness will be selected.

\subsubsection{The Crossover of the HGA}

This paper presents a new crossover operator, Best Insertion Crossover (BIC), which combines neighborhood insertion algorithm with greedy algorithm. The insertion mechanism is limited by vehicle capacity and resource point time window to generate viable offspring while minimizing route cost (including total time, distance and risk).

BIC consists of two steps, that is, deleting and inserting. The deletion part first selects two parents, $\mathrm{P} 1$ and P2 randomly. Secondly, a sub-path is selected in P1 and P2 respectively, and then the requirement points in the sub-path selected by another parent are deleted from the solution of the current parent. Insertion is the insertion of missing nodes into the descendants. The insertion rules are as follows:

Step1, inserts the demand point $\mathrm{R}$ sequentially at the insertion point. The insertion point refers to the interval between any adjacent demand points in the current routing. In the insertion process, vehicle capacity constraints and time window constraints must be met to generate viable offspring.

Step2, calculate the cost after each insertion. Find the best insertion point of demand point $\mathrm{R}$ at the lowest cost.

Step3, if all the points do not satisfy constraints, a new sub-path can be constructed.

\subsubsection{The Mutation of the HGA}

The exchange mutation is used as a mutation method, in which two genes are randomly exchanged in a sub-path, as shown in Figure 2. In the mutation process, the demand points 4 and 1 of sub-path 1 are exchanged.

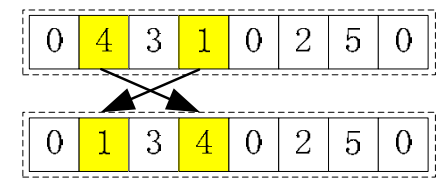

Fig. 2 The exchange mutation

\section{The Experimental Results and Discussions}

Assuming that there are three resource points, 150 request points, $\mathrm{x}$, y position of demand point $\mathrm{n}$ and the demand number of equipment $\mathrm{v}$ for demand point $\mathrm{n}$ are known, assuming that the load of each vehicle is 10 tons, and the transport vehicle travels at a uniform speed of $70 \mathrm{~km} / \mathrm{h}$ on each road. The traffic jam on each road and the influence factor of weather conditions are 0.2 and 0.3 , respectively. The support level of the three resource points is $0.3,0.2$ and 0.5 respectively, and the loading time of equipment $\mathrm{v}$ unit is $0.05 \mathrm{~h} / \mathrm{t}$. 
Firstly, allocate the request points to the resources. Figure 3 is a schematic diagram of 150 demand points, in which the red point represents the demand point allocated to resource point 1 , the yellow point represents the demand point allocated to resource point 2, and the blue point represents the demand point allocated to resource point 3 . From the graph, we can see that the demand points are allocated to the nearest resource points centrally.

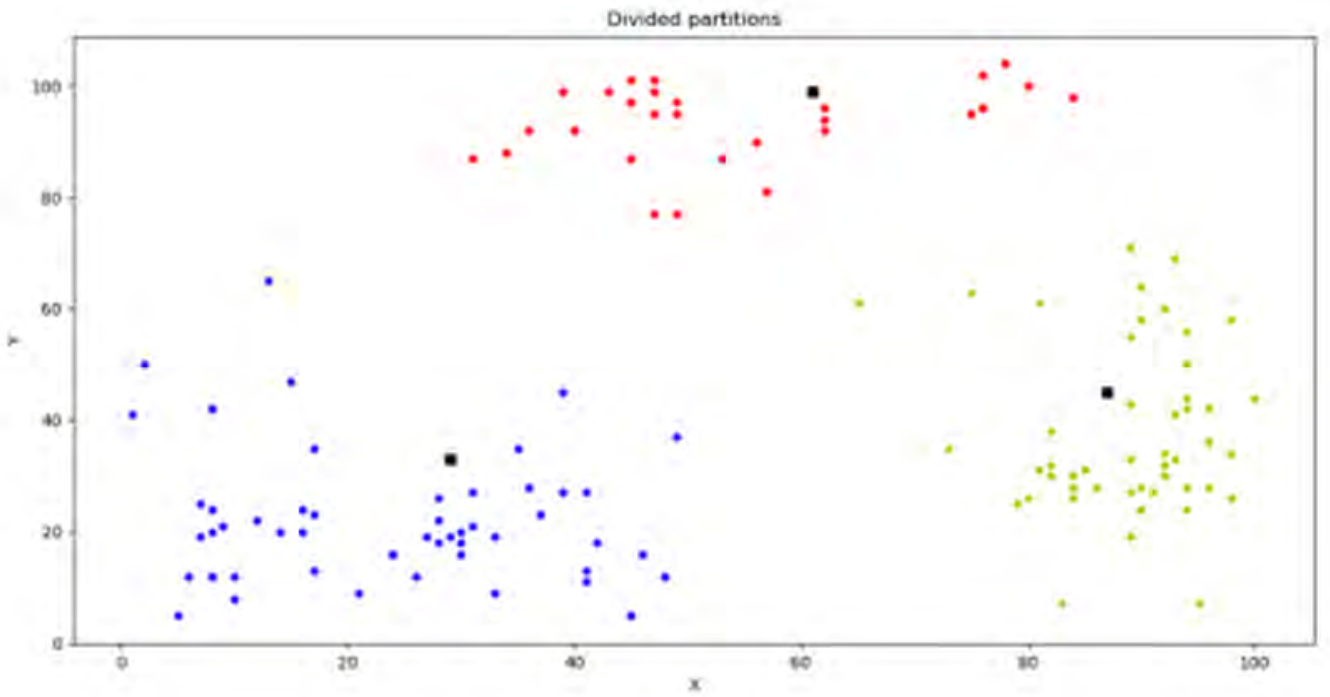

Fig.3 The allocation of the request points

HGA and simulated annealing-based particle swarm optimization (SAPSO) are used to solve the path planning problem in equipment distribution. The population size of HGA is 100, the number of iterations is 150 , the crossover probability is 0.8 , and the mutation probability is 0.1 . The number of SAPSO particles is 500, the number of iterations is 300 , the learning factor $\mathrm{c} 1$ and $\mathrm{c} 2$ are 2.05 , and the annealing constant is 0.5 . Each algorithm runs 30 times, and the best delivery plan is shown in Figure 4. In addition, figure 5 shows the total support time and cost of these two methods.

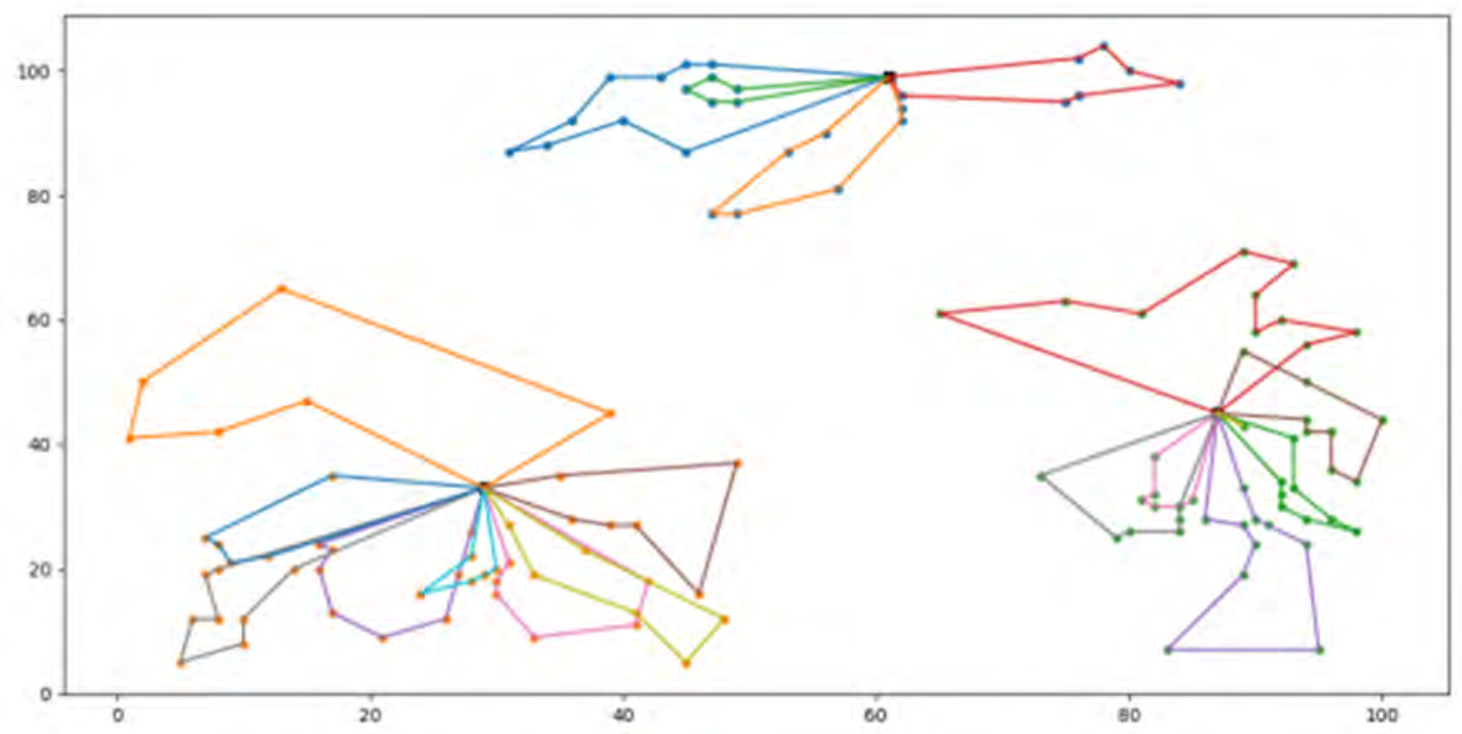

(a) The solution of HGA 


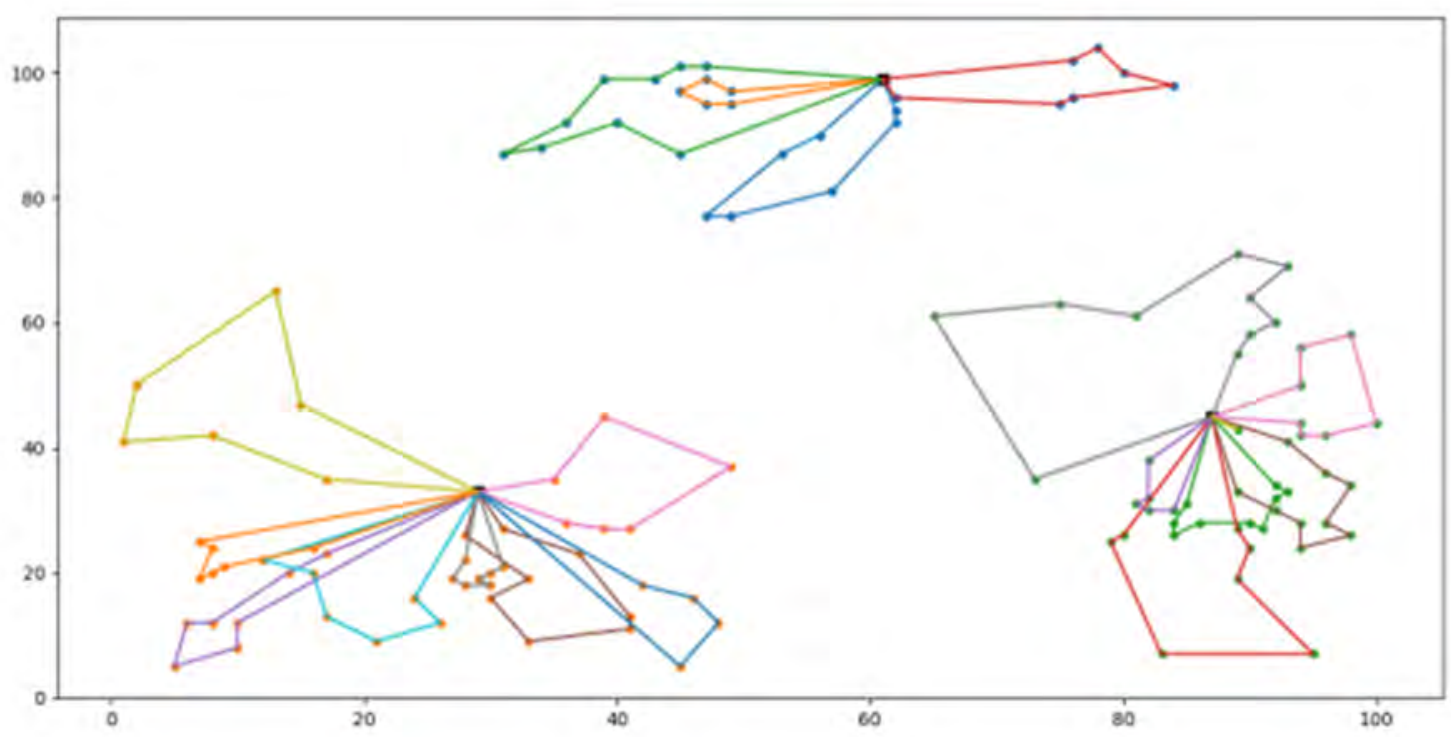

(b) The solution of SAPSO

Fig.4 The solution of the material assignment

Table 1. The total support time and cost of HGA and SAPSO

\begin{tabular}{ccccc}
\hline & \multicolumn{3}{c}{ HGA } & \multicolumn{2}{c}{ SAPSO } \\
\hline & Total time & Total cost & Total time & Total cost \\
Summation & 65.37 & 4482 & 65.62 & 4570 \\
\hline
\end{tabular}

The objective function of the equipment distribution problem is to minimize the total delivery time and the guarantee cost. It can be seen that the HGA solution distribution scheme is superior to SAPSO in the total delivery time and the guarantee cost, indicating that the HGA algorithm performs better in solving the equipment allocation problem.

\section{Summary}

This paper studies the optimization of maintenance equipment distribution in material equipment support decision-making. Firstly, the multi-objective optimization model of maintenance equipment distribution problem is constructed, including minimizing the total delivery time and guarantee cost. The maintenance equipment distribution problem is further divided into two phases: (i) demand point allocation and (ii) path optimization within a single resource point. The former divides the demand point into the resource points of the nearest distance, thus transforming the multi-vehicle vehicle routing problem (MDVRP) problem into the ordinary VRP problem. Secondly, we propose hybrid genetic algorithm (HGA) to deal with the routing problem in a single resource point. The validity of the two algorithms is verified by a construction case based on three resource points and 150 demand points. Experiments show that HGA outperforms SAPSO in solving equipment allocation problems.

\section{Acknowledgments}

Fund project: Equipment field pre-research fund project: Equipment Autonomic Logistics Decision and System Architecture Design Technology (61400040501).

\section{References}

[1]. Maozhi Gan, Jianshe Kang, Qi Gao. Military Equipment Maintenance Engineering [M]. Beijing: National Defense Industry Press. 
[2]. Zhiwei Shan. Equipment Comprehensive Support Engineering [M]. Beijing: National Defense Industry Press, 2007.

[3]. Yunchun Jiang, Jing Qiu, Junrong Pan. Research on the Concept and System of Equipment Autonomy Maintenance Support[J]. China Mechanical Engineering. 2004, 15(5): 402-40.

[4]. S. Wang, J. Watada, A hybrid modified PSO approach to VaR-based facility location problems with variable capacity in fuzzy random uncertainty, Inf. Sci. (Ny).192 (2012) 3-18.

[5]. S. Wang, J. Watada, W. Pedrycz, Value-at-risk-based two-stage fuzzy facility location problems, IEEE Trans. Ind. Inform. 5 (2009) 465-482.

[6]. S. Wang, J. Watada, W. Pedrycz, Recourse-based facility-location problems in hybrid uncertain environment, IEEE Trans. Syst. Man Cybern. Part B: Cybern.40 (2010) 1176-1187.

[7]. Y.-J. Gong, J. Zhang, O. Liu, R.-Z. Huang, H.S.-H. Chung, Y.-H. Shi, Optimizing the vehicle routing problem with time windows: a discrete particle swarm opti-mization approach, IEEE Trans. Syst. Man Cybern. Part C: Appl. Rev. 42 (2012)254-267.

[8]. S.H.R. Pasandideh, S.T.A. Niaki, Genetic application in a facility location problem with random demand within queuing framework, J. Intell. Manuf. 23 (2010)651-659.

[9]. J. Qin, L. Ni, F. Shi, Combined simulated annealing algorithm for the discrete facility location problem, Sci. World J. 2012 (2012) 1-7.

[10]. V.F. Yu, S.-W. Lin, W. Lee, C.-J. Ting, A simulated annealing heuristic for the capacitated location routing problem, Comput. Ind. Eng. 58 (2010) 288-299.

[11]. M. Sun, A tabu search heuristic procedure for the capacitated facility location problem, J. Heuristics 18 (2011) 91-118.

[12]. J.-F. Cordeau, M. Maisch Berger, A parallel iterated tabu search heuristic for vehicle routing problems, Comput. Oper. Res. 39 (2012) 2033-2050. 